\title{
Pointwise estimates for a class of non-homogeneous Kolmogorov equations
}

\author{
Chiara Cinti · Andrea Pascucci · Sergio Polidoro
}

Received: 18 December 2006

(C) Springer-Verlag 2007

\begin{abstract}
We consider a class of ultraparabolic differential equations that satisfy the Hörmander's hypoellipticity condition and we prove that the weak solutions to the equation with measurable coefficients are locally bounded functions. The method extends the Moser's iteration procedure and has previously been employed in the case of operators verifying a further homogeneity assumption. Here we remove that assumption by proving some potential estimates and some ad hoc Sobolev type inequalities for solutions.
\end{abstract}

Mathematics Subject Classification (2000) $35 \mathrm{~K} 57 \cdot 35 \mathrm{~K} 65 \cdot 35 \mathrm{~K} 70$

\section{Introduction}

We consider a class of second order partial differential equations of KolmogorovFokker-Planck type with measurable coefficients in the form

$L u(x, t):=\sum_{i, j=1}^{m_{0}} \partial_{x_{i}}\left(a_{i j}(x, t) \partial_{x_{j}} u(x, t)\right)+\sum_{i, j=1}^{N} b_{i j} x_{i} \partial_{x_{j}} u(x, t)-\partial_{t} u(x, t)=0$

where $(x, t)=\left(x_{1}, \ldots, x_{N}, t\right)=z$ denotes the point in $\mathbb{R}^{N+1}$, and $1 \leq m_{0} \leq N$.

C. Cinti · A. Pascucci · S. Polidoro $(\varangle)$

Dipartimento di Matematica, Università di Bologna,

Piazza di Porta S. Donato 5, 40126 Bologna, Italy

e-mail: polidoro@dm.unibo.it

C. Cinti

e-mail: cinti@dm.unibo.it

A. Pascucci

e-mail: pascucci@dm.unibo.it 
In order to state our assumptions, we define the principal part of $L$ as follows

$$
K=\Delta_{m_{0}}+Y
$$

where $\Delta_{m_{0}}$ is the Laplace operator in the variables $x_{1}, \ldots, x_{m_{0}}$ and $Y$ is the first order part of $L$ :

$$
Y=\sum_{i, j=1}^{N} b_{i j} x_{i} \partial_{x_{j}}-\partial_{t}
$$

Our assumptions are:

[H.1] the principal part $K$ of $L$ is hypoelliptic (i.e., every distributional solution of $K u=0$ is a $C^{\infty}$ function);

[H.2] the coefficients $a_{i j}, 1 \leq i, j \leq m_{0}$, are real valued, measurable functions of $z$. Moreover $a_{i j}=a_{j i}, 1 \leq i, j \leq m_{0}$, and there exists a positive constant $\mu$ such that

$$
\mu^{-1}|\xi|^{2} \leq \sum_{i, j=1}^{m_{0}} a_{i j}(z) \xi_{i} \xi_{j} \leq \mu|\xi|^{2}
$$

for every $z \in \mathbb{R}^{N+1}$ and $\xi \in \mathbb{R}^{m_{0}}$. The matrix $B=\left(b_{i j}\right)_{i, j=1, \ldots, N}$ is constant.

In the sequel, an equation of the form (1) satisfying [H.1]-[H.2] will be simply called a KFP equation. A well-known criterion for the hypoellipticity of $K$ is the Hörmander's condition [16] which in our setting reads:

$$
\operatorname{rank} \operatorname{Lie}\left(\partial_{x_{1}}, \ldots, \partial_{x_{m_{0}}}, Y\right)(z)=N+1, \quad \forall z \in \mathbb{R}^{N+1}
$$

where Lie $\left(\partial_{x_{1}}, \ldots, \partial_{x_{m_{0}}}, Y\right)$ denotes the Lie algebra generated by the first order differential operators (vector fields) $\partial_{x_{1}}, \ldots, \partial_{x_{m_{0}}}, Y$. Then [H.1] only depends on $m_{0}$ and on the first order part of $L$. We explicitly remark that uniformly parabolic operators (for which $m_{0}=N$ and $B \equiv 0$ ) are KFP operators and the related principal part is the usual heat operator in $\mathbb{R}^{N+1}$. On the other hand, there are also several examples of degenerate KFP operators, i.e., with $m_{0}$ strictly lesser than $N$, from diffusion theory and mathematical finance.

Example 1 Consider the following kinetic equation

$$
\partial_{t} f-\left\langle v, \nabla_{x} f\right\rangle=\mathcal{Q}(f), \quad t \geq 0, x \in \mathbb{R}^{n}, v \in \mathbb{R}^{n}
$$

where $n \geq 1$ and $\mathcal{Q}(f)$ is the so-called "collision operator" which can take either a linear or a non-linear form. The solution $f$ corresponds at each time $t$ to the density of particles at the point $x$ with velocity $v$. If

$$
\mathcal{Q}(f)=\operatorname{div}_{v}\left(\nabla_{v} f+v f\right),
$$


then (4) becomes the prototype of the linear Fokker-Planck equation (see, for instance, [8] and [32]) and it can be written in the form (1) by choosing $m_{0}=n, N=2 n$ and

$$
B=\left(\begin{array}{cc}
I_{n} & I_{n} \\
0 & 0
\end{array}\right),
$$

where $I_{n}$ is the identity $n \times n$ matrix.

In the Boltzmann-Landau equation (see $[6,20]$ and [21])

$$
\mathcal{Q}(f)=\sum_{i, j=1}^{n} \partial_{v_{i}}\left(a_{i j}(\cdot, f) \partial_{v_{j}} f\right)
$$

the coefficients $a_{i j}$ depend on the unknown function through some integral expression.

Example 2 Equations of the form (1) arise in mathematical finance as well. More specifically, the following linear KFP equation

$$
S^{2} \partial_{S S} V+f(S) \partial_{M} V-\partial_{t} V=0, \quad S, t>0, M \in \mathbb{R}
$$

with either $f(S)=\log S$ or $f(S)=S$, arises in the Black and Scholes theory when considering the problem of the pricing of Asian options (see [3]). Moreover, in the stochastic volatility model by Hobson and Rogers, the price of an European option is given as a solution of the KFP equation

$$
\frac{1}{2} \sigma^{2}(S-M)\left(\partial_{S S} V-\partial_{S} V\right)+(S-M) \partial_{M} V-\partial_{t} V=0,
$$

for some positive continuous function $\sigma$ (see [15] and [9]). In the theory of bonds and interest rates, KFP equations are considered in the study of the possible realization of Heath-Jarrow-Morton [14] models in terms of a finite dimensional Markov diffusion (see, for instance, [33] and [4]). We finally recall that non-linear KFP equations of the form

$$
\Delta_{x} u+h(u) \partial_{y} u-\partial_{t} u=f(\cdot, u), \quad(x, y, t) \in \mathbb{R}^{m_{0}} \times \mathbb{R} \times \mathbb{R} .
$$

occur in the theory of stochastic utility theory (see [1,2], and [7]).

It is well known that the natural geometric setting for the study of KFP operators is the analysis on Lie groups (see for instance [13,34]).

The theory has been widely developed in the simplest case of homogeneous Lie groups. A systematic study of this class of operators, when the coefficents $a_{i j}$ are constant, has been carried out by Kupcov [18], and by Lanconelli and Polidoro [19]. The existence of a fundamental solution has been proved by Weber [36], Il'in [17], Eidelman [12] and Polidoro [29,30] in the case of Hölder continuous coefficients $a_{i j}$. Pointwise upper and lower bound for the fundamental solution, mean value formulas and Harnack inequalities are given in [29,30]; Schauder type estimates have been proved by Satyro [35], Lunardi [22], Manfredini [23]. 
In the more general case of non-homogeneous groups, the existence of a fundamental solution has been proved in [19] for KFP operators with constant coefficients and by Di Francesco and Pascucci in [10] for Hölder continuous coefficients. We also recall some mean value formulas proved by Morbidelli in [25] and Harnack type inequalities in [25] and in [11].

Concerning the regularity of the weak solutions to (1), we recall the papers $[5,24$, 31 , where the coefficients $a_{i j}$ satisfy a suitable vanishing mean oscillation condition. In [28] we proved some pointwise estimate for the weak solutions to (1) by adapting a classical iterative method introduced by Moser [26,27] to the non-Euclidean framework of the homogeneous Lie groups. The main goal in the papers by Moser is a Harnack inequality. With the aim to adapt this theory to operators in the form (1), a crucial step is the proof of a Poincaré type inequality which has not yet been established.

The Moser's method is based on a combination of a Caccioppoli type estimate with the classical embedding Sobolev inequality. Due to the strong degeneracy of the KFP operators, we encountered in [28] a new difficulty: the natural extension of the Caccioppoli estimates gives an $L_{\text {loc }}^{2}$ bound only of the first order derivatives $\partial_{x_{1}} u, \ldots, \partial_{x_{m_{0}}} u$ of the solution $u$ of (1), but it does not give any information on the other spatial directions. The main idea used in [28] is to prove a Sobolev type inequality only for the solutions to (1), by using a representation formula for the solution $u$ in terms of the fundamental solution of the principal part $K$ of $L$. More specifically, let $u$ be a solution to (1), then

$$
K u=(K-L) u=\sum_{i=1}^{m_{0}} \partial_{x_{i}} F_{i},
$$

where

$$
F_{i}=\sum_{j=1}^{m_{0}}\left(\delta_{i j}-a_{i j}\right) \partial_{x_{j}} u, \quad i=1, \ldots, m_{0}
$$

Since the $F_{i}$ 's depend only on the first order derivatives $\partial_{x_{j}} u, j=1, \ldots, m_{0}$, the Caccioppoli inequality yields an $H_{\text {loc }}^{-1}$-estimate of the right hand side of (5). Thus, by using some potential estimate for the fundamental solution of $K$, we prove the needed bound for the $L_{\text {loc }}^{p}$ norm of $u$.

The proof of the Caccioppoli type inequality plainly extends to non-homogeneous groups, whereas the Sobolev inequalities used in [28] heavily rely on the homogeneity of the fundamental solution. The main results of this paper are some $L^{p}$ potential estimates for the convolution with the non-homogeneous fundamental solution $\Gamma$ of $K$ and with the derivatives $\partial_{x_{1}} \Gamma, \ldots, \partial_{x_{m_{0}}} \Gamma$, that are given in Sect. 3, Theorem 2. Section 2 contains some known facts about $K$ and on the related Lie group. Section 4 is devoted to the Moser's iterative procedure.

In order to state our main results we introduce some notations. We denote by $D=\left(\partial_{x_{1}}, \ldots, \partial_{x_{N}}\right),\langle\cdot, \cdot\rangle$, respectively, the gradient and the inner product in $\mathbb{R}^{N}$. Besides, $D_{m_{0}}$ is the gradient with respect to the variables $x_{1}, \ldots, x_{m_{0}}$. We also write 
operator $L$ in (1) and the vector field $Y$ defined in (3) in a more compact form:

$$
L=\operatorname{div}(A D)+Y, \quad Y=\langle x, B D\rangle-\partial_{t}
$$

where $A=\left(a_{i j}\right)_{1 \leq i, j \leq N}, a_{i j} \equiv 0$ if $i>m_{0}$ or $j>m_{0}$.

Definition 1 A weak solution of (1) in a subset $\Omega$ of $\mathbb{R}^{N+1}$ is a function $u$ such that $u, D_{m_{0}} u, Y u \in L_{\mathrm{loc}}^{2}(\Omega)$ and

$$
\int_{\Omega}-\langle A D u, D \varphi\rangle+\varphi Y u=0, \quad \forall \varphi \in C_{0}^{\infty}(\Omega) .
$$

In the sequel we will also consider weak sub-solutions of (1), namely functions $u$ such that $u, D_{m_{0}} u, Y u \in L_{\text {loc }}^{2}(\Omega)$ and

$$
\int_{\Omega}-\langle A D u, D \varphi\rangle+\varphi Y u \geq 0, \quad \forall \varphi \in C_{0}^{\infty}(\Omega), \varphi \geq 0 .
$$

Moreover $u$ is a weak super-solution of (1) if $-u$ is a sub-solution. Clearly, if $u$ is a sub and super-solution of (1), then it is a solution.

As we shall see in Sect. 2, the natural geometry underlying operator $L$ is determined by a suitable homogeneous Lie group structure on $\mathbb{R}^{N+1}$. Our main results below reflect this non-Euclidean background. Let "o" denote the Lie product on $\mathbb{R}^{N+1}$ defined in (13), and consider the cylinder

$$
R_{1}=\left\{(x, t) \in \mathbb{R}^{N} \times \mathbb{R}|| x|<1,| t \mid<1\right\}
$$

For every $z_{0} \in \mathbb{R}^{N+1}$ and $r>0$, we set

$$
R_{r}\left(z_{0}\right) \equiv z_{0} \circ \delta_{r}\left(R_{1}\right)=\left\{z \in \mathbb{R}^{N+1} \mid z=z_{0} \circ \delta_{r}(\zeta), \zeta \in R_{1}\right\}
$$

We also denote $R_{r}=R_{r}(0)$. Our main result is the following

Theorem 1 Let $u$ be a non-negative weak solution of (1) in $\Omega$. Let $z_{0} \in \Omega$ and $r, \varrho$, $0<\frac{r}{2} \leq \varrho<r \leq 1$, be such that $\overline{R_{r}\left(z_{0}\right)} \subseteq \Omega$. Then there exists a positive constant $c$ which depends on $\mu$ and on the homogeneous dimension $Q$ (cf. (21)) such that, for 
every $p>0$, it holds

$$
\sup _{R_{\varrho}\left(z_{0}\right)} u^{p} \leq \frac{c}{(r-\varrho)^{Q+2}} \int_{R_{r}\left(z_{0}\right)} u^{p} .
$$

Estimate (10) also holds for every $p<0$ such that $u^{p} \in L^{1}\left(R_{r}\left(z_{0}\right)\right)$.

Remark 1 Sub and super-solutions also verify estimate (10) for suitable values of $p$ (see Corollary 2). More precisely, (10) holds for

(i) $p \geq 1$ or $p<0$, if $u$ is a non-negative weak sub-solution of (1) such that $u^{p} \in L^{1}\left(R_{r}\left(z_{0}\right)\right)$;

(ii) $\quad p \in] 0, \frac{1}{2}[$, if $u$ is a non-negative weak super-solution of (1). In this case, the constant $c$ in (10) also depends on $p$.

A direct consequence of Theorem 1 is the local boundedness of weak solutions to (1).

Corollary 1 Let $u$ be a weak solution of (1) in $\Omega$. Let $z_{0}, \varrho, r$ as in Theorem 1 . Then, we have

$$
\sup _{R_{\varrho}\left(z_{0}\right)}|u| \leq\left(\frac{c}{(r-\varrho)^{Q+2}} \int_{R_{r}\left(z_{0}\right)}|u|^{p}\right)^{\frac{1}{p}}, \quad \forall p \geq 1
$$

where $c=c(Q, \mu)$.

The following result restores the analogy with the classical result by Moser. Denote $R_{r}^{-}\left(x_{0}, t_{0}\right)=R_{r}\left(x_{0}, t_{0}\right) \cap\left\{t<t_{0}\right\}$, then

Proposition 1 Let $u$ be a non-negative weak solution of (1) in $\Omega$. Let $z_{0} \in \Omega$ and $r, \varrho, 0<\frac{r}{2} \leq \varrho<r \leq 1$, be such that $\overline{R_{r}^{-}\left(z_{0}\right)} \subseteq \Omega$. Suppose that $u^{p} \in L^{1}\left(R_{r}^{-}\left(z_{0}\right)\right)$, for some $p<0$. Then there exists a positive constant $c$ which depends on $\mu$ and on the homogeneous dimension $Q$ such that

$$
\sup _{R_{\varrho}^{-}\left(z_{0}\right)} u^{p} \leq \frac{c}{(r-\varrho)^{Q+2}} \int_{R_{r}^{-}\left(z_{0}\right)} u^{p} .
$$

\section{Preliminaries}

In this section we recall some known facts about the principal part $K$ of $L$, and we give some preliminary results. We first recall that $K$ is invariant with respect to a Lie product in $\mathbb{R}^{N+1}$. More specifically, we let

$$
E(s)=\exp \left(-s B^{T}\right), \quad s \in \mathbb{R},
$$


and we denote by $\ell_{\zeta}, \zeta \in \mathbb{R}^{N+1}$, the left translation $\ell_{\zeta}(z)=\zeta \circ z$ in the group law

$$
(x, t) \circ(\xi, \tau)=(\xi+E(\tau) x, t+\tau), \quad(x, t),(\xi, \tau) \in \mathbb{R}^{N+1},
$$

then we have

$$
K \circ \ell_{\zeta}=\ell_{\zeta} \circ K
$$

We recall that, by Proposition 2.1 of [19], hypothesis [H.1] is equivalent to assume that for some basis on $\mathbb{R}^{N}$, the matrix $B$ has the canonical form

$$
\left(\begin{array}{ccccc}
* & B_{1} & 0 & \cdots & 0 \\
* & * & B_{2} & \cdots & 0 \\
\vdots & \vdots & \vdots & \ddots & \vdots \\
* & * & * & \cdots & B_{r} \\
* & * & * & \cdots & *
\end{array}\right)
$$

where $B_{k}$ is a $m_{k-1} \times m_{k}$ matrix of rank $m_{k}, k=1,2, \ldots, r$ with

$$
m_{0} \geq m_{1} \geq \ldots m_{r} \geq 1, \quad \text { and } \quad \sum_{k=0}^{r} m_{k}=N
$$

and the blocks denoted by " $*$ " are arbitrary.

We denote by $\Gamma(\cdot, \zeta)$ the fundamental solution of $K$ in (2) with pole at $\zeta \in \mathbb{R}^{N+1}$. An explicit expression of $\Gamma(\cdot, \zeta)$ has been constructed in [16] and [18]:

$$
\Gamma(z, \zeta)=\Gamma\left(\zeta^{-1} \circ z, 0\right), \quad \forall z, \zeta \in \mathbb{R}^{N+1}, z \neq \zeta
$$

where

$$
\Gamma((x, t),(0,0))= \begin{cases}\frac{(4 \pi)^{-\frac{N}{2}}}{\sqrt{\operatorname{det} \mathcal{C}(t)}} \exp \left(-\frac{1}{4}\left\langle\mathcal{C}^{-1}(t) x, x\right\rangle-t \operatorname{tr}(B)\right) & \text { if } t>0 \\ 0 & \text { if } t \leq 0\end{cases}
$$

and

$$
\mathcal{C}(t)=\int_{0}^{t} E(s)\left(\begin{array}{cc}
I_{m_{0}} & 0 \\
0 & 0
\end{array}\right) E^{T}(s) d s
$$

$\left(E(\cdot)\right.$ is the matrix defined in (12) and $I_{m_{0}}$ is the $m_{0} \times m_{0}$ identity matrix). Note that hypothesis [H.1] implies that $\mathcal{C}(t)$ is strictly positive for every positive $t$ (see [19, Proposition A.1]). If we denote by $K^{*}$ the formal adjoint of $K: K^{*}=\Delta_{m_{0}}+Y^{*}$, and by $\Gamma^{*}$ its fundamental solution, then

$$
\Gamma^{*}(z, \zeta)=\Gamma(\zeta, z), \quad \text { for every } z, \zeta \in \mathbb{R}^{N+1}: z \neq \zeta
$$


Let us explicitly note that, since $Y^{*}=-Y-\operatorname{tr} B$, we have

$$
\int_{\mathbb{R}^{N}} \Gamma^{*}(x, t, \xi, 0) d \xi=e^{t \operatorname{tr} B}, \quad \text { for every }(x, t) \in \mathbb{R}^{N} \times \mathbb{R}^{+} .
$$

Let $K_{0}=\Delta_{m_{0}}+Y_{0}$ be an operator satisfying condition [H.1], where $Y_{0}=$ $\left\langle x, B_{0} D\right\rangle-\partial_{t}$ and

$$
B_{0}=\left(\begin{array}{ccccc}
0 & B_{1} & 0 & \cdots & 0 \\
0 & 0 & B_{2} & \cdots & 0 \\
\vdots & \vdots & \vdots & \ddots & \vdots \\
0 & 0 & 0 & \cdots & B_{r} \\
0 & 0 & 0 & \cdots & 0
\end{array}\right)
$$

Then $K_{0}$ is invariant with respect to the dilations defined as

$$
\delta_{\lambda}=\operatorname{diag}\left(\lambda I_{m_{0}}, \lambda^{3} I_{m_{1}}, \ldots, \lambda^{2 r+1} I_{m_{r}}, \lambda^{2}\right)=\operatorname{diag}\left(D_{\lambda}, \lambda^{2}\right), \quad \lambda>0,
$$

where $I_{m_{k}}$ denotes the $m_{k} \times m_{k}$ identity matrix. More specifically (see [19, Proposition 2.2]) we have that

$$
K_{0} \circ \delta_{\lambda}=\lambda^{2}\left(\delta_{\lambda} \circ K_{0}\right), \text { for every } \lambda>0 \text {. }
$$

In (19) "o" denotes the composition law related to $K_{0}$. The converse implication is also true: $K$ is invariant with respect to the dilations $\left(\delta_{\lambda}\right)_{\lambda>0}$ if, and only if, the $*$-blocks of $B$ in (14) are zero matrices. In that case the corresponding matrices $E_{0}$ and $\mathcal{C}_{0}^{-1}$ satisfy

$$
E_{0}\left(\lambda^{2} s\right)=D_{\lambda} E_{0}(s) D_{\frac{1}{\lambda}}, \quad \mathcal{C}_{0}^{-1}\left(\lambda^{-2} t\right)=D_{\lambda} \mathcal{C}_{0}^{-1}(t) D_{\lambda}
$$

for any $s, t \in \mathbb{R}$ and $\lambda>0$. The fundamental solution $\Gamma_{0}$ of $K_{0}$ is a homogeneous function with respect to $\left(\delta_{\lambda}\right)_{\lambda>0}$, namely

$$
\Gamma_{0}\left(\delta_{\lambda}(z), 0\right)=\lambda^{-Q} \Gamma_{0}(z, 0), \quad \text { for every } z \in \mathbb{R}^{N+1} \backslash\{0\}, \lambda>0,
$$

where

$$
Q=m_{0}+3 m_{1}+\cdots+(2 r+1) m_{r} .
$$

We denote by $\|\cdot\|$ the following norm:

$$
\|z\| \equiv\left(\sum_{j=1}^{N} x_{j}^{\alpha_{j}}+|t|^{\frac{(2 r+1) !}{2}}\right)^{\frac{1}{(2 r+1) !}}
$$


where $\alpha_{j}=(2 r+1)$ ! if $1 \leq j \leq m_{0}$ and

$$
\alpha_{j}=\frac{(2 r+1) !}{2 k+1}, \quad \text { if } 1+\sum_{i=0}^{k-1} m_{i} \leq j \leq \sum_{i=0}^{k} m_{i}, 1 \leq k \leq r
$$

Note that $\|\cdot\|$ is $\delta_{\lambda}$-homogeneous of degree 1, i.e.,

$$
\left\|\delta_{\lambda} z\right\|=\lambda\|z\| \text { for every } \lambda>0
$$

We will denote by

$$
\mathcal{B}(\zeta, \varrho)=\left\{z \in \mathbb{R}^{N+1}:\left\|\zeta^{-1} \circ z\right\|<\varrho\right\}
$$

the ball with center at $\zeta \in \mathbb{R}^{N+1}$ and radius $\varrho>0$. As we will see in Sect. 3 [formula (47)] we have

$$
\text { meas } \mathcal{B}(\zeta, \varrho)=\varrho^{Q+2} \text { meas } \mathcal{B}(0,1)
$$

("meas" denotes the Lebesgue measure) then the natural number $Q+2$ will be called the homogeneous dimension of $\mathbb{R}^{N+1}$ with respect to $\left(\delta_{\lambda}\right)_{\lambda>0}$.

It is known that homogeneous operators provide a good approximation of the nonhomogeneous ones. In order to be more specific, consider any operator $K=\Delta_{m_{0}}+$ $\langle x, B D\rangle-\partial_{t}$ satisfying condition [H.1]. Define $K_{0}=\Delta_{m_{0}}+\left\langle x, B_{0} D\right\rangle-\partial_{t}$, where $B_{0}$ is the matrix in (17), and denote by $\Gamma_{0}$ its fundamental solution of $K_{0}$. Then, for every $b>0$, there exists a positive constant $a$ such that

$$
\frac{1}{a} \Gamma_{0}(z) \leq \Gamma(z) \leq a \Gamma_{0}(z)
$$

for every $z \in \mathbb{R}^{N+1}$ such that $\Gamma_{0}(z) \geq b$ (see [19, Theorem 3.1]). The above result says that, in some sense, $\Gamma_{0}$ shares some homogeneity properties with $\Gamma$, so that we can use the norm $\|\cdot\|$ also when $K$ is not invariant with respect to $\left(\delta_{\lambda}\right)_{\lambda>0}$. We explicitly note that the dilations $\left(\delta_{\lambda}\right)_{\lambda>0}$ only depend on the matrix $B_{0}$.

For every $\lambda \in] 0,1]$ we set

$$
K_{\lambda}=\lambda^{2}\left(\delta_{\lambda} \circ K \circ \delta_{\frac{1}{\lambda}}\right)
$$

In order to explicitly write $K_{\lambda}$ and its fundamental solution, we note that, if

$$
B=\left(\begin{array}{ccccc}
B_{0,0} & B_{1} & 0 & \cdots & 0 \\
B_{1,0} & B_{1,1} & B_{2} & \cdots & 0 \\
\vdots & \vdots & \vdots & \ddots & \vdots \\
B_{r-1,0} & B_{r-1,1} & B_{r-1,2} & \cdots & B_{r} \\
B_{r, 0} & B_{r, 1} & B_{r, 2} & \cdots & B_{r, r}
\end{array}\right)
$$


where $B_{i, j}$ are the $m_{i} \times m_{j}$ blocks denoted by "*" in (14), then $K_{\lambda}=\Delta_{m_{0}}+Y_{\lambda}$, where

$$
Y_{\lambda}:=\left\langle x, B_{\lambda} D\right\rangle-\partial_{t}
$$

and $B_{\lambda}:=\lambda^{2} D_{\lambda} B D_{\frac{1}{\lambda}}$, i.e.,

$$
B_{\lambda}=\left(\begin{array}{ccccc}
\lambda^{2} B_{0,0} & B_{1} & 0 & \cdots & 0 \\
\lambda^{4} B_{1,0} & \lambda^{2} B_{1,1} & B_{2} & \cdots & 0 \\
\vdots & \vdots & \vdots & \ddots & \vdots \\
\lambda^{2 r} B_{r-1,0} & \lambda^{2 r-2} B_{r-1,1} & \lambda^{2 r-4} B_{r-1,2} & \cdots & B_{r} \\
\lambda^{2 r+2} B_{r, 0} & \lambda^{2 r} B_{r, 1} & \lambda^{2 r-2} B_{r, 2} & \cdots & \lambda^{2} B_{r, r}
\end{array}\right)
$$

The fundamental solution $\Gamma_{\lambda}$ of $K_{\lambda}$ is given by

$$
\Gamma_{\lambda}((x, t),(0,0))= \begin{cases}\frac{(4 \pi)^{-\frac{N}{2}}}{\sqrt{\operatorname{det} \mathcal{C}_{\lambda}(t)}} \exp \left(-\frac{1}{4}\left\langle\mathcal{C}_{\lambda}^{-1}(t) x, x\right\rangle-t \operatorname{tr}\left(B_{\lambda}\right)\right) & \text { if } t>0 \\ 0 & \text { if } t \leq 0\end{cases}
$$

with

$$
E_{\lambda}(s)=\exp \left(-s B_{\lambda}^{T}\right), \quad \mathcal{C}_{\lambda}(t)=\int_{0}^{t} E_{\lambda}(s)\left(\begin{array}{cc}
I_{m_{0}} & 0 \\
0 & 0
\end{array}\right) E_{\lambda}^{T}(s) d s
$$

Since the translation group related to $K_{\lambda}$ depends on $\lambda$, it will be denoted by " ${ }_{\lambda}$ ":

$$
(x, t) \circ_{\lambda}(\xi, \tau)=\left(\xi+E_{\lambda}(\tau) x, t+\tau\right), \quad(x, t),(\xi, \tau) \in \mathbb{R}^{N+1} .
$$

We remark explicitly that $o_{\lambda}$ defines a 1-parameter family of Lie groups structures, in which $\lambda=0$ corresponds to a homogeneous Lie group structure.

We recall that, for every given $T>0$, there exists a positive constant $c_{T}$ such that

$$
\begin{aligned}
& \left\langle\mathcal{C}_{0}(t) x, x\right\rangle\left(1-c_{T} \lambda^{2} t\right) \leq\left\langle\mathcal{C}_{\lambda}(t) x, x\right\rangle \leq\left\langle\mathcal{C}_{0}(t) x, x\right\rangle\left(1+c_{T} \lambda^{2} t\right) \\
& \left\langle\mathcal{C}_{0}^{-1}(t) y, y\right\rangle\left(1-c_{T} \lambda^{2} t\right) \leq\left\langle\mathcal{C}_{\lambda}^{-1}(t) y, y\right\rangle \leq\left\langle\mathcal{C}_{0}^{-1}(t) y, y\right\rangle\left(1+c_{T} \lambda^{2} t\right)
\end{aligned}
$$

for every $\left.\left.x, y \in \mathbb{R}^{N}, t \in\right] 0, T\right]$ and $\lambda \in[0,1]$ (see [19, Formula (3.23) and (3.24)]). In the sequel we will also use the following result

Lemma 1 Let $T>0$ and $c_{T}$ as above. Then:

i) there exists a positive constant $c_{T}^{\prime}$ such that

$$
\left\|D_{\sqrt{t}}\left(\mathcal{C}_{\lambda}^{-1}(t)-\mathcal{C}_{0}^{-1}(t)\right) D_{\sqrt{t}}\right\| \leq c_{T}^{\prime} \lambda^{2} t
$$

for every $t \in] 0, T]$ and $\lambda \in[0,1]$; 
ii) there exist two positive constants $c_{T}^{\prime \prime}, c_{T}^{\prime \prime \prime}$ such that

$$
c_{T}^{\prime \prime} t^{Q}\left(1-c_{T} \lambda^{2} t\right) \leq \operatorname{det} \mathcal{C}_{\lambda}(t) \leq c_{T}^{\prime \prime \prime} t^{Q}\left(1+c_{T} \lambda^{2} t\right),
$$

for every $\left.\left.(x, t) \in \mathbb{R}^{N} \times\right] 0, T\right]$ and $\lambda \in[0,1]$ such that $t<\frac{1}{c_{T}}$.

Proof i) Since $\mathcal{C}_{\lambda}^{-1}(t)$ and $\mathcal{C}_{0}^{-1}(t)$ are symmetric, we have

$$
\begin{gathered}
\left\|D_{\sqrt{t}}\left(\mathcal{C}_{\lambda}^{-1}(t)-\mathcal{C}_{0}^{-1}(t)\right) D_{\sqrt{t}}\right\|=\sup _{|y| \leq 1}\left\langle\left(\mathcal{C}_{\lambda}^{-1}(t)-\mathcal{C}_{0}^{-1}(t)\right) D_{\sqrt{t}} y, D_{\sqrt{t}} y\right\rangle \\
\leq c_{T} \lambda^{2} t \sup _{|y| \leq 1}\left\langle\mathcal{C}_{0}^{-1}(t) D_{\sqrt{t}} y, D_{\sqrt{t}} y\right\rangle=c_{T} \lambda^{2} t \sup _{|y| \leq 1}\left\langle\mathcal{C}_{0}^{-1}(1) y, y\right\rangle
\end{gathered}
$$

by the second set of inequalities in (30) and the second identity in (20). This proves the claim.

ii) Let $\mu_{k}$ be the $k$-th eigenvalue of $D_{\frac{1}{\sqrt{t}}} \mathcal{C}_{\lambda}(t) D_{\frac{1}{\sqrt{t}}}$, and let $v_{k}$ be one of the corresponding eigenvector; it is not restrictive to assume that $\left|v_{k}\right|=1$. Then (30) yields

$$
\left\langle\mathcal{C}_{0}(t) D_{\frac{1}{\sqrt{t}}} v_{k}, D_{\frac{1}{\sqrt{t}}} v_{k}\right\rangle\left(1-c_{T} \lambda^{2} t\right) \leq \mu_{k} \leq\left\langle\mathcal{C}_{0}(t) D_{\frac{1}{\sqrt{t}}} v_{k}, D_{\frac{1}{\sqrt{t}}} v_{k}\right\rangle\left(1+c_{T} \lambda^{2} t\right)
$$

so that, by (20),

$$
\left\langle\mathcal{C}_{0}(1) v_{k}, v_{k}\right\rangle\left(1-c_{T} \lambda^{2} t\right) \leq \mu_{k} \leq\left\langle\mathcal{C}_{0}(1) v_{k}, v_{k}\right\rangle\left(1+c_{T} \lambda^{2} t\right)
$$

Since $\operatorname{det}\left(D_{\frac{1}{\sqrt{t}}} \mathcal{C}_{\lambda}(t) D_{\frac{1}{\sqrt{t}}}\right)$ is the product of its eigenvalues, from the above inequality it follows that

$$
c_{T}^{\prime \prime}\left(1-c_{T} \lambda^{2} t\right)^{N} \leq \operatorname{det}\left(D_{\frac{1}{\sqrt{t}}} \mathcal{C}_{\lambda}(t) D_{\frac{1}{\sqrt{t}}}\right) \leq c_{T}^{\prime \prime \prime}\left(1+c_{T} \lambda^{2} t\right)^{N},
$$

for suitable positive constants $c_{T}^{\prime \prime}, c_{T}^{\prime \prime \prime}$, provided that $t$ is suitable small. Thus, (ii) follows from the fact that $\operatorname{det} D_{\sqrt{t}}=t^{\frac{Q}{2}}$.

\section{Potential estimates}

In this section we prove some $L^{q}$ estimates of the $\Gamma_{\lambda}$-potential of a function $f \in$ $L^{p}\left(\mathbb{R}^{N+1}\right)$ :

$$
\Gamma_{\lambda}(f)(z):=\int_{\mathbb{R}^{N+1}} \Gamma_{\lambda}(z, \zeta) f(\zeta) d \zeta, \quad z \in \mathbb{R}^{N+1}
$$


We will also consider the potential $\Gamma_{\lambda}\left(D_{m_{0}} f\right)$, i.e.,

$$
\Gamma_{\lambda}\left(D_{m_{0}} f\right)(z):=-\int_{\mathbb{R}^{N+1}} D_{m_{0}}^{(\zeta)} \Gamma_{\lambda}(z, \zeta) f(\zeta) d \zeta
$$

where $D_{m_{0}} \Gamma_{\lambda}(x, t, \xi, \tau)$ is the gradient with respect to $x_{1}, \ldots, x_{m_{0}}$ and the superscript in $D_{m_{0}}^{(\zeta)}$ indicates that we are differentiating w.r.t. the variable $\zeta$.

The main result of this section is the following $L^{p}$ estimate in the domain $S_{T}=$ $\left.\left.\mathbb{R}^{N} \times\right] 0, T\right]$.

Theorem 2 Let $f \in L^{2}\left(S_{T}\right)$. There exists a positive constant $c=c(T, B)$ such that

$$
\begin{aligned}
\left\|\Gamma_{\lambda}(f)\right\|_{L^{2 \widetilde{\kappa}}\left(S_{T}\right)} & \leq c\|f\|_{L^{2}\left(S_{T}\right)}, \\
\left\|\Gamma_{\lambda}\left(D_{m_{0}} f\right)\right\|_{L^{2 \kappa}\left(S_{T}\right)} & \leq c\|f\|_{L^{2}\left(S_{T}\right)},
\end{aligned}
$$

for every $\lambda \in] 0,1]$, where $\widetilde{\kappa}=1+\frac{4}{Q-2}$ and $\kappa=1+\frac{2}{Q}$.

We first prove an uniform (in $\lambda$ ) pointwise bound for $\Gamma_{\lambda}$ and $D_{m_{0}}^{(\zeta)} \Gamma_{\lambda}$.

Proposition 2 For every $T>0$ there exists a positive constant $C_{T}$ such that:

$$
\begin{aligned}
\Gamma_{\lambda}(z, \zeta) & \leq \frac{C_{T}}{\left\|\zeta^{-1} \circ_{\lambda} z\right\|^{Q}}, \\
\left|D_{m_{0}}^{(\zeta)} \Gamma_{\lambda}(z, \zeta)\right| & \leq \frac{C_{T}}{\left\|\zeta^{-1} \circ_{\lambda} z\right\|^{Q+1}}
\end{aligned}
$$

for every $z, \zeta \in S_{T}$ and $\left.\left.\lambda \in\right] 0,1\right]$.

Proof Let $z=(x, t), \zeta=(\xi, \tau) \in S_{T}$ and $\left.\left.\lambda \in\right] 0,1\right]$. Denote $w=(y, s)=\zeta^{-1} \circ_{\lambda} z=$ $\left(x-E_{\lambda}(t-\tau) \xi, t-\tau\right)$. Then, in order to prove (35), it is sufficient to show that

$$
\left.\left.\|w\|^{Q} \Gamma_{\lambda}(w) \leq C_{T}, \quad \text { for every } w \in S_{T} \text { and } \lambda \in\right] 0,1\right]
$$

By (27) and Lemma 1 we get

$$
\Gamma_{\lambda}(y, s) \leq \frac{(4 \pi)^{-\frac{N}{2}}}{\sqrt{c_{T}^{\prime \prime} Q_{\left(1-c_{T} \lambda^{2} s\right)}}} e^{-\frac{1}{4}\left\langle\mathcal{C}_{0}^{-1}(s) y, y\right\rangle\left(1-c_{T} \lambda^{2} s\right)-\lambda^{2} s \operatorname{tr}(B)}
$$

for $s<\frac{1}{c_{T}}$. On the other hand, (20) yields

$$
\left\langle\mathcal{C}_{0}^{-1}(s) y, y\right\rangle=\left\langle\mathcal{C}_{0}^{-1}(1) D_{\frac{1}{\sqrt{s}}} y, D_{\frac{1}{\sqrt{s}}} y\right\rangle \geq\left|D_{\frac{1}{\sqrt{s}}} y\right|^{2} \min _{|\eta|=1}\left\langle\mathcal{C}_{0}^{-1}(1) \eta, \eta\right\rangle
$$


and (22) gives

$$
\|(y, s)\|=\left\|\left(D_{\sqrt{s}} D_{\frac{1}{\sqrt{s}}} y, s\right)\right\|=\sqrt{s}\left\|\left(D_{\frac{1}{\sqrt{s}}} y, 1\right)\right\| \leq \widetilde{c} \sqrt{s}\left(\left|D_{\frac{1}{\sqrt{s}}} y\right|+1\right)
$$

for a constant $\tilde{c}$ only dependent on the norm. Hence,

$$
\|(y, s)\|^{Q} \Gamma_{\lambda}(y, s) \leq C_{0}\left(\left|D_{\frac{1}{\sqrt{s}}} y\right|+1\right)^{Q} \exp \left(-c_{0}\left|D_{\frac{1}{\sqrt{s}}} y\right|^{2}\right)
$$

for every $(y, s) \in S_{T}$ such that $s<\frac{1}{2 c_{T}}$, where the constants $C_{0}, c_{0}$ only depend on $T$ and on the matrix $B$. This proves the claim (37) for $s<\frac{1}{2 c_{T}}$.

If $\frac{1}{2 c_{T}}>T$ the proof is accomplished, otherwise we have to show that (37) holds in the set $\mathbb{R}^{N} \times\left[\frac{1}{2 c_{T}}, T\right]$. To that aim, we observe that $\operatorname{det} \mathcal{C}_{\lambda}(s)$ is a positive function which continuously depend on $(s, \lambda)$ in the compact set $\left[\frac{1}{2 c_{T}}, T\right] \times[0,1]$. The same assertion holds for the positive matrix $D_{\sqrt{s}} \mathcal{C}_{\lambda}^{-1}(s) D_{\sqrt{s}}$. Then

$$
\Gamma_{\lambda}(y, s) \leq C_{1} \exp \left(-\frac{c_{1}}{4}\left|D_{\frac{1}{\sqrt{s}}} y\right|^{2}\right)
$$

where

$$
\begin{gathered}
C_{1}=\frac{(4 \pi)^{-\frac{N}{2}} e^{T|\operatorname{tr}(B)|}}{\sqrt{\min _{\left[\frac{1}{2 c_{T}}, T\right] \times[0,1]} \operatorname{det} \mathcal{C}_{\lambda}(s)}}, \\
c_{1}=\min _{\left[\frac{1}{2 c_{T}}, T\right] \times[0,1]} \min _{|\eta|=1}\left\langle D_{\sqrt{s}} \mathcal{C}_{\lambda}^{-1}(s) D_{\sqrt{s}} \eta, \eta\right\rangle .
\end{gathered}
$$

Then, by using again (38), we get

$$
\|(y, s)\|^{Q} \Gamma_{\lambda}(y, s) \leq C_{1} s^{\frac{Q}{2}} \widetilde{c}^{Q}\left(\left|D_{\frac{1}{\sqrt{s}}} y\right|+1\right)^{Q} \exp \left(-\frac{c_{1}}{4}\left|D_{\frac{1}{\sqrt{s}}} y\right|^{2}\right)
$$

for every $(y, s) \in \mathbb{R}^{N} \times\left[\frac{1}{2 c_{T}}, T\right]$. This proves that

$$
\|(y, s)\|^{Q} \Gamma_{\lambda}(y, s) \leq C_{2}\left(\left|D_{\frac{1}{\sqrt{s}}} y\right|+1\right)^{Q} \exp \left(-c_{2}\left|D_{\frac{1}{\sqrt{s}}} y\right|^{2}\right)
$$

for every $(y, s) \in S_{T}$, where the constants $C_{2}, c_{2}$ only depend on $T$ and on the matrix $B$. Since the right hand side of (40) is a bounded function, we get the claim (37). 
In order to simplify the proof of (36), we first observe that (16) implies

$$
D_{m_{0}}^{(\zeta)} \Gamma_{\lambda}(z, \zeta)=D_{m_{0}} \Gamma_{\lambda}^{*}(\zeta, z)
$$

so that it is sufficient to consider $\partial_{\xi_{j}} \Gamma_{\lambda}^{*}(\xi, \tau, x, t)$ for $j=1, \ldots, m_{0}$.

As before, we let $(\eta, \sigma)=z^{-1} \circ_{\lambda} \zeta=\left(\xi-E_{\lambda}(\tau-t) x, \tau-t\right)$ and we note that

$$
\partial_{\eta_{j}} \Gamma_{\lambda}^{*}(\eta, \sigma)=-\frac{1}{2} \Gamma_{\lambda}^{*}(\eta, \sigma)\left(\mathcal{C}_{\lambda}^{-1}(-\sigma) \eta\right)_{j} \quad \text { for } j=1, \ldots, m_{0}
$$

We next claim that

$$
\left|\left(\mathcal{C}_{\lambda}^{-1}(-\sigma) \eta\right)_{j}\right| \leq \frac{C_{3}}{\sqrt{-\sigma}}\left|D_{\frac{1}{\sqrt{-\sigma}}} \eta\right| \text { for } j=1, \ldots, m_{0}
$$

for every $(\eta, \sigma) \in \mathbb{R}^{N} \times\left[-T, 0\left[\right.\right.$, where the constant $C_{3}$ only depends on $T$ and on the matrix $B$, so that, by (38), we obtain

$$
\|(\eta, \sigma)\| \cdot\left|\left(\mathcal{C}_{\lambda}^{-1}(-\sigma) \eta\right)_{j}\right| \leq \widetilde{c} C_{3}\left(\left|D_{\frac{1}{\sqrt{-\sigma}} \eta}\right|+1\right)^{2}
$$

On the other hand, the same argument used in the proof of (40) gives the following estimate

$$
\|(\eta, \sigma)\|^{Q} \Gamma_{\lambda}^{*}(\eta, \sigma) \leq C_{2}\left(\left|D_{\frac{1}{\sqrt{-\sigma}}} \eta\right|+1\right)^{Q} \exp \left(-c_{2}\left|D_{\frac{1}{\sqrt{-\sigma}}} \eta\right|^{2}\right)
$$

for every $(\eta, \sigma) \in \mathbb{R}^{N} \times[-T, 0[$, and (36) follows from (43) and (41).

We next prove (42):

$$
\begin{aligned}
\left|\left(\mathcal{C}_{\lambda}^{-1}(-\sigma) \eta\right)_{j}\right| \leq & \left|\left(\left(\mathcal{C}_{\lambda}^{-1}(-\sigma)-\mathcal{C}_{0}^{-1}(-\sigma)\right) \eta\right)_{j}\right|+\left|\left(\mathcal{C}_{0}^{-1}(-\sigma) \eta\right)_{j}\right| \\
= & \frac{1}{\sqrt{-\sigma}}\left|\left(D_{\sqrt{-\sigma}}\left(\mathcal{C}_{\lambda}^{-1}(-\sigma)-\mathcal{C}_{0}^{-1}(-\sigma)\right) D_{\sqrt{-\sigma}} D_{\frac{1}{\sqrt{-\sigma}} \eta}\right)_{j}\right| \\
& +\frac{1}{\sqrt{-\sigma}}\left|\left(D_{\sqrt{-\sigma}} \mathcal{C}_{0}^{-1}(-\sigma) D_{\sqrt{-\sigma}} D_{\frac{1}{\sqrt{-\sigma}} \eta}\right)_{j}\right| \\
\leq & \frac{1}{\sqrt{-\sigma}}\left|D_{\sqrt{-\sigma}}\left(\mathcal{C}_{\lambda}^{-1}(-\sigma)-\mathcal{C}_{0}^{-1}(-\sigma)\right) D_{\sqrt{-\sigma}} \| \cdot\right| D_{\frac{1}{\sqrt{-\sigma}} \eta} \eta \mid \\
& +\frac{1}{\sqrt{-\sigma}}\left|\mathcal{C}_{0}^{-1}(1) D_{\frac{1}{\sqrt{-\sigma}}} \eta\right|,
\end{aligned}
$$


by (20). From Lemma $1-(i)$ it follows that

$$
\left|\left(\mathcal{C}_{\lambda}^{-1}(-\sigma) \eta\right)_{j}\right| \leq \frac{1}{\sqrt{-\sigma}}\left(c_{T}^{\prime} \lambda^{2}(-\sigma)+C_{4}\right)\left|D_{\frac{1}{\sqrt{-\sigma}} \eta} \eta\right|
$$

where $C_{4}$ depends on $\mathcal{C}_{0}^{-1}(1)$. This proves (42).

In view of Proposition 2 we define, for $\lambda \in[0,1], \alpha \in] 0, Q+2[$ and $p>1$

$$
I_{\lambda}^{\alpha} f(z)=\left(\operatorname{meas} \mathcal{B}_{\lambda}(0,1)\right)^{-\frac{\alpha}{Q+2}} \int_{S_{T} \cap\{\tau<t\}} \frac{f(\zeta)}{\left\|\zeta^{-1} \circ_{\lambda} z\right\|^{\alpha}} d \zeta, \quad z \in \mathbb{R}^{N+1}
$$

where $f \in L^{p}\left(S_{T}\right)$. We next prove a result which is analogous to the classical potential estimates on homogeneous Lie groups (cf., for instance, [13]).

Proposition 3 Let $\alpha \in] 0, Q+2\left[, \lambda \in[0,1]\right.$ and $T>0$. Consider $f \in L^{p}\left(S_{T}\right)$ for some $p \in] 1,+\infty\left[\right.$. Then the function $I_{\lambda}^{\alpha} f$ is defined almost everywhere and there exists a constant $c=c(T, B, p, \alpha)$ such that

$$
\left\|I_{\lambda}^{\alpha} f\right\|_{L^{q}\left(S_{T}\right)} \leq c\|f\|_{L^{p}\left(S_{T}\right)},
$$

where $q$ is defined by

$$
\frac{1}{q}=\frac{1}{p}+\frac{\alpha}{Q+2}-1
$$

The proof is analogous to that in the framework of homogeneous Lie groups: the main difference occurs in the change of variable of integration, where some extra terms appear.

Remark 2 Let $T \in \mathbb{R}^{+}, \lambda \in[0,1]$. For any $f \in L^{1}\left(S_{T}\right)$ we have

$$
\begin{aligned}
\int_{\left.\mathbb{R}^{N} \times\right] 0, t[} f\left(\zeta^{-1} \circ_{\lambda} z\right) d \zeta & =\int_{\left.\mathbb{R}^{N} \times\right] 0, t[} e^{s \lambda^{2} \operatorname{tr} B} f(y, s) d y d s, \quad \forall z=(x, t) \in S_{T} ; \\
\int_{\left.\mathbb{R}^{N} \times\right] \tau, T[} f\left(\zeta^{-1} \circ_{\lambda} z\right) d z & =\int_{\left.\mathbb{R}^{N} \times\right] 0, T-\tau[} f(y, s) d y d s, \quad \forall \zeta=(\xi, \tau) \in S_{T} .
\end{aligned}
$$

Indeed, it suffices to perform the change of variable $\Phi(y, s)=\left(E_{\lambda}(-s)(x-y), t-s\right)$ in the first integral and note that $\operatorname{det} E_{\lambda}(-s)=e^{s \lambda^{2} \operatorname{tr} B}$, by (26) and (28). On the other hand, in the second integral we use the change of variable $\Psi(y, s)=\left(y+E_{\lambda}(s) \xi, \tau+s\right)$, and note that $\operatorname{det} J_{\Psi}(w)=1$.

In particular, the second identity in (46) yields that for every ball

$$
\mathcal{B}_{\lambda}(\zeta, \varrho)=\left\{z \in \mathbb{R}^{N+1}:\left\|\zeta^{-1} \circ_{\lambda} z\right\|<\varrho\right\}
$$


it holds

$$
\text { meas } \mathcal{B}_{\lambda}(\zeta, \varrho)=\varrho^{Q+2} \text { meas } \mathcal{B}_{\lambda}(0,1)
$$

We next prove a Young type inequality for the inhomogeneous Lie group related to $K_{\lambda}$. Note that the Lie group corresponding to $\lambda=0$ is homogeneous and Lemma 2 restores the standard Young inequality.

Lemma 2 Let $p, q, r \in[1, \infty]$ be three constant such that $\frac{1}{p}+\frac{1}{q}=1+\frac{1}{r}$ and let $\lambda \in[0,1]$. Let $f \in L^{p}\left(S_{T}\right)$ and $g \in L^{q}\left(S_{T}\right)$, then the function $f * \lambda g$ defined as

$$
f *_{\lambda} g(z)=\int_{S_{T} \cap\{\tau<t\}} f\left(\zeta^{-1} \circ_{\lambda} z\right) g(\zeta) d \zeta
$$

belongs to $L^{r}\left(S_{T}\right)$ and

$$
\left\|f *_{\lambda} g\right\|_{L^{r}\left(S_{T}\right)} \leq e^{\lambda^{2} T\left(1-\frac{1}{q}\right)|\operatorname{tr}(B)|}\|f\|_{L^{p}\left(S_{T}\right)}\|g\|_{L^{q}\left(S_{T}\right)} .
$$

Proof We argue as in the proof of the classical Young's inequality, and use Remark 2. For any $\alpha, \beta \in[0,1]$ and $p_{1}, p_{2} \geq 0$ such that $\frac{1}{p_{1}}+\frac{1}{p_{2}}+\frac{1}{r}=1$ we have

$$
\begin{aligned}
\left|f *_{\lambda} g(z)\right| \leq & \int_{S_{T} \cap\{\tau<t\}}\left|f\left(\zeta^{-1} \circ \lambda z\right)\right| g(\zeta) \mid d \zeta \\
\leq & \left(\int_{S_{T} \cap\{\tau<t\}}\left|f\left(\zeta^{-1} \circ_{\lambda} z\right)\right|^{(1-\alpha) r}|g(\zeta)|^{(1-\beta) r} d \zeta\right)^{\frac{1}{r}} \\
& \cdot\left(\int_{S_{T} \cap\{\tau<t\}}\left|f\left(\zeta^{-1} \circ_{\lambda} z\right)\right|^{\alpha p_{1}} d \zeta\right)^{\frac{1}{p_{1}}} \\
& \cdot\left(\int_{S_{T} \cap\{\tau<t\}}|g(\zeta)|^{\beta p_{2}} d \zeta\right)^{\frac{1}{p_{2}}}
\end{aligned}
$$

by the Hölder inequality. We then change variable in the last but one integral: by Remark 2 we have

$$
\int_{S_{T} \cap\{\tau<t\}}\left|f\left(\zeta^{-1} \circ \lambda z\right)\right|^{\alpha p_{1}} d \zeta \leq e^{T \lambda^{2}|\operatorname{tr} B|} \int_{\left.\mathbb{R}^{N} \times\right] 0, t[}|f(w)|^{\alpha p_{1}} d w
$$


Thus, by integrating in the set $S_{T}$, we get

$$
\begin{aligned}
\|f * \lambda g\|_{L^{r}\left(S_{T}\right)} \leq & e^{\frac{T \lambda^{2}|\operatorname{tr} B|}{p_{1}}}\|f\|_{L^{\alpha p_{1}\left(S_{T}\right)}}^{\alpha}\|g\|_{L^{\beta p_{2}\left(S_{T}\right)}}^{\beta} \\
& \cdot\left(\int_{S_{T}}|g(\zeta)|^{(1-\beta) r}\left(\int_{S_{T} \cap\{t>\tau\}}\left|f\left(\zeta^{-1} \circ_{\lambda} z\right)\right|^{(1-\alpha) r} d z\right) d \zeta\right)^{\frac{1}{r}} .
\end{aligned}
$$

We change again the variable in the last integral: in this case Remark 2 gives

$$
\begin{gathered}
\left\|f *_{\lambda} g\right\|_{L^{r}\left(S_{T}\right) \leq} e^{\frac{T \lambda^{2}|\operatorname{tr} B|}{p_{1}}}\|f\|_{L^{\alpha p_{1}\left(S_{T}\right)}}^{\alpha}\|f\|_{L^{(1-\alpha) r}\left(S_{T}\right)}^{1-\alpha} \\
\cdot\|g\|_{L^{\beta p_{2}\left(S_{T}\right)}}^{\beta}\|g\|_{L^{(1-\beta) r}\left(S_{T}\right)}^{1-\beta}
\end{gathered}
$$

From this point we conclude the proof as in the classical case: for any given $p, q, r \in$ $[1, \infty]$ such that $\frac{1}{p}+\frac{1}{q}=1+\frac{1}{r}$ we choose $\alpha=1-\frac{p}{r}, \beta=1-\frac{q}{r}$ (note that $\alpha, \beta \in[0,1]$ ), then $p_{1}=\frac{p}{\alpha}, p_{2}=\frac{q}{\beta}$ (so that $\alpha p_{1}=(1-\alpha) r$ and $\beta p_{2}=(1-\beta) r$ ). The proof of the Proposition then follows from (48).

Proof of Proposition 3 As in the proof of Lemma 2, we follow a classical argument and use Remark 2 when it is needed.

We first introduce some standard notation. Consider a measurable function $f$ : $\Omega \rightarrow \mathbb{R}$ where $\Omega$ denotes a measurable subset of $\mathbb{R}^{N+1}$, and let $\beta_{f}(a)=\operatorname{meas}\{z \in$ $\Omega:|f(z)|>a\}$ denote its distribution function. We say that $f$ belongs to the space $L_{\mathrm{w}}^{p}(\Omega)$ (for $p \geq 1$ ) if there exists a positive constant $C$ such that $\beta_{f}(a) \leq\left(\frac{C}{a}\right)^{p}$, for every positive $a$. In that case

$$
\|f\|_{L_{\mathrm{w}}^{p}(\Omega)}=\inf \left\{C>0: \beta_{f}(a) \leq\left(\frac{C}{a}\right)^{p}\right\}
$$

is the weak- $L^{p}$ norm of $f$. We also recall that

$$
\|f\|_{L^{p}(\Omega)}=\left(p \int_{0}^{\infty} a^{p-1} \beta_{f}(a) d a\right)^{\frac{1}{p}} .
$$

In order to prove (45), we show that, for every $p, q \in] 1, \infty\left[\right.$ satisfying $\frac{1}{q}+1=$ $\frac{1}{p}+\frac{\alpha}{Q+2}$, we have

$$
\left\|I_{\lambda}^{\alpha} f\right\|_{L_{\mathrm{w}}^{q}\left(S_{T}\right)} \leq \bar{C}\|f\|_{L^{p}\left(S_{T}\right)}
$$

for a positive constant $\bar{C}$ depending on $T, \alpha, p$ and on the matrix $B$. The thesis follows from the Marcinkiewicz interpolation theorem. 
In order to simplify the proof of (50), we assume $\|f\|_{L^{p}\left(S_{T}\right)}=1$, since it is not restrictive. Moreover we write Eq. (44) as

$$
I_{\lambda}^{\alpha} f(z)=\int_{S_{T} \cap\{\tau<t\}} g_{\alpha}\left(\zeta^{-1} \circ_{\lambda} z\right) f(\zeta) d \zeta
$$

where

$$
g_{\alpha}(w)=\frac{\left(\text { meas } \mathcal{B}_{\lambda}(0,1)\right)^{-\frac{\alpha}{Q+2}}}{\|w\|^{\alpha}} .
$$

Note that, by (47), $g_{\alpha}$ has norm equal to one in the space $L_{\mathrm{w}}^{\frac{Q+2}{\alpha}}\left(\mathbb{R}^{N+1}\right)$.

For any $a>0$, we set

$$
b=\left(\frac{a}{2}\left(\frac{Q+2}{q \alpha}\right)^{\frac{p-1}{p}} e^{-\frac{p-1}{p} \lambda^{2} T|\operatorname{tr} B|}\right)^{\frac{q \alpha}{Q+2}}
$$

we define

$$
g_{\alpha}^{+}(w)=\left\{\begin{array}{ll}
g_{\alpha}(w), & \text { if } g_{\alpha}(w)>b, \\
0, & \text { otherwise, }
\end{array} \quad g_{\alpha}^{-}(w)=g_{\alpha}(w)-g_{\alpha}^{+}(w),\right.
$$

and

$$
\begin{aligned}
& J_{\alpha}^{+} f(z)=\int_{S_{T} \cap\{\tau<t\}} g_{\alpha}^{+}\left(\zeta^{-1} \circ_{\lambda} z\right) f(\zeta) d \zeta, \\
& J_{\alpha}^{-} f(z)=\int_{S_{T} \cap\{\tau<t\}} g_{\alpha}^{-}\left(\zeta^{-1} \circ_{\lambda} z\right) f(\zeta) d \zeta .
\end{aligned}
$$

To prove (50), we recall (51) and note that

$$
\beta_{I_{\lambda}^{\alpha} f}(a) \leq \beta_{J_{\alpha}^{+} f}(a / 2)+\beta_{J_{\alpha}^{-} f}(a / 2)
$$

We first consider the term $\beta_{J_{\alpha}^{-} f}$. By the Hölder inequality we get

$$
\begin{aligned}
& \left|J_{\alpha}^{-} f(z)\right| \leq\left(\int_{S_{T} \cap\{\tau<t\}}\left|g_{\alpha}^{-}\left(\zeta^{-1} \circ_{\lambda} z\right)\right|^{\frac{p}{p-1}} d \zeta\right)^{\frac{p-1}{p}}\|f\|_{L^{p}\left(S_{T}\right)} \\
& \leq e^{\lambda^{2} T \frac{p-1}{p}|\operatorname{tr} B|}\left(\int_{S_{T} \cap\{\tau<t\}}\left|g_{\alpha}^{-}(y, s)\right|^{\frac{p}{p-1}} d y d s\right)^{\frac{p-1}{p}},
\end{aligned}
$$


by Remark 2, since we assume $\|f\|_{L^{p}\left(S_{T}\right)}=1$. By using (49) and (52) we find

$$
\left(\int_{S_{T} \cap\{\tau<t\}}\left|g_{\alpha}^{-}(y, s)\right|^{\frac{p}{p-1}} d y d s\right)^{\frac{p-1}{p}} \leq \frac{a}{2} e^{-\lambda^{2} T \frac{p-1}{p}|\operatorname{tr} B|},
$$

so that $\left|J_{\alpha}^{-} f(z)\right| \leq \frac{a}{2}$ for every $z \in S_{T}$. Thus

$$
\beta_{J_{\alpha}^{-} f}(a / 2)=0
$$

We next consider the term $\beta_{J_{\alpha}^{+} f}$. By Lemma 2 we have

$$
\begin{aligned}
\left\|J_{\alpha}^{+} f\right\|_{L^{p}\left(S_{T}\right)} & \leq e^{\lambda^{2} T\left(1-\frac{1}{p}\right)|\operatorname{tr}(B)|}\left\|g_{\alpha}^{+}\right\|_{L^{1}\left(S_{T}\right)}\|f\|_{L^{p}\left(S_{T}\right)} \\
& \leq e^{\lambda^{2} T\left(1-\frac{1}{p}\right)|\operatorname{tr}(B)|} \frac{\alpha}{Q+2-\alpha} b^{1-\frac{Q+2}{\alpha}}
\end{aligned}
$$

since $\|f\|_{L^{p}\left(S_{T}\right)}=1$ and

$$
\left\|g_{\alpha}^{+}\right\|_{L^{1}\left(S_{T}\right)} \leq \frac{\alpha}{Q+2-\alpha} b^{1-\frac{Q+2}{\alpha}}
$$

by (49). Thus, being $\left\|J_{\alpha}^{+} f\right\|_{L_{\mathrm{w}}^{p}\left(S_{T}\right)} \leq\left\|J_{\alpha}^{+} f\right\|_{L^{p}\left(S_{T}\right)}$, we get

$$
\beta_{J_{\alpha}^{+} f}(a / 2) \leq a^{-p} e^{\lambda^{2} T(p-1)|\operatorname{tr}(B)|}\left(\frac{2 \alpha}{Q+2-\alpha}\right)^{p} b^{p\left(1-\frac{Q+2}{\alpha}\right)}=\bar{C} a^{-q}
$$

where the $\bar{C}$ is a positive constant that depends on $T, \alpha, p$ and on the matrix $B$ (recall our choice (52) of $b$ ). Then the above inequality and (54) give

$$
\beta_{I_{\lambda}^{\alpha} f}(a) \leq \bar{C} a^{-q}
$$

for any $a>0$. This proves (50) and concludes the proof.

Proof of Theorem 2 By Proposition 2 we get

$$
\begin{aligned}
\left|\Gamma_{\lambda}(f)(z)\right| & \leq \bar{C}_{T} I_{\lambda}^{Q}|f(z)|, \\
\left|\Gamma_{\lambda}\left(D_{m_{0}} f\right)(z)\right| & \leq \bar{C}_{T} I_{\lambda}^{Q+1}|f(z)|,
\end{aligned}
$$

for every $z \in S_{T}$. Estimates (33) and (34) then follow from Proposition 3.

As in the homogeneous case (see [28, Lemma 2.5]) we can use the fundamental solution $\Gamma$ as a test function in the definition of sub and super-solution. 
Lemma 3 Let $v$ be a weak sub-solution of $\operatorname{div}(A D v)+Y_{\lambda} v=0$ in $\Omega$. For every $\varphi \in C_{0}^{\infty}(\Omega), \varphi \geq 0$, and for almost every $z \in \mathbb{R}^{N+1}$, we have

$$
\int_{\Omega}-\left\langle A D v, D\left(\Gamma_{\lambda}(z, \cdot) \varphi\right)\right\rangle+\Gamma_{\lambda}(z, \cdot) \varphi Y_{\lambda} v \geq 0 .
$$

An analogous result holds for weak super-solutions.

Proof For every $\varepsilon>0$, we set

$$
\chi_{\varepsilon}(z, \zeta)=\chi\left(\frac{\left\|\zeta^{-1} \circ z\right\|}{\varepsilon}\right), \quad z, \zeta \in \mathbb{R}^{N+1},
$$

where $\chi \in C^{1}([0,+\infty[,[0,1])$ is such that $\chi(s)=0$ for $s \in[0,1], \chi(s)=1$ for $s \geq 2$ and $0 \leq \chi^{\prime} \leq 2$. By (8), for every $\varepsilon>0$ and $z \in \mathbb{R}^{N+1}$, we have

$$
\begin{aligned}
0 & \leq \int_{\Omega}-\left\langle A D v, D\left(\Gamma_{\lambda}(z, \cdot) \chi_{\varepsilon}(z, \cdot) \varphi\right)\right\rangle+\Gamma_{\lambda}(z, \cdot) \chi_{\varepsilon}(z, \cdot) \varphi Y_{\lambda} v \\
& =-I_{1, \varepsilon}(z)+I_{2, \varepsilon}(z)-I_{3, \varepsilon}(z)
\end{aligned}
$$

where

$$
\begin{aligned}
& I_{1, \varepsilon}(z)=\int_{\Omega}\left\langle A D v, D\left(\Gamma_{\lambda}(z, \cdot)\right)\right\rangle \chi_{\varepsilon}(z, \cdot) \varphi, \\
& I_{2, \varepsilon}(z)=\int_{\Omega} \Gamma_{\lambda}(z, \cdot) \chi_{\varepsilon}(z, \cdot)\left(-\langle A D v, D \varphi\rangle+\varphi Y_{\lambda} v\right), \\
& I_{3, \varepsilon}(z)=\int_{\Omega}\left\langle A D v, D \chi_{\varepsilon}(z, \cdot)\right\rangle \Gamma_{\lambda}(z, \cdot) \varphi .
\end{aligned}
$$

Consider the first integral. Since $\varphi \chi_{\varepsilon}(z, \cdot) A D v \rightarrow \varphi A D v$ in $L^{2}\left(S_{T}\right)$, as $\varepsilon \rightarrow 0$, (34) of Theorem 2 gives

$$
I_{1, \varepsilon}(z) \rightarrow \int_{\Omega}\left\langle A D v, D\left(\Gamma_{\lambda}(z, \cdot)\right)\right\rangle \varphi
$$

as $\varepsilon \rightarrow 0$, for almost every $z \in S_{T}$. The same argument applies to the second and third integrals, by (33) and, since $I_{3, \varepsilon}(z) \rightarrow 0$ as $\varepsilon \rightarrow 0$, we conclude the proof.

We next state, without proof, the following

Lemma 4 Let $f \in C^{2} \cap \operatorname{Lip}(\mathbb{R})$ be a monotone non-decreasing function. If $f$ is convex (resp. concave) and $u$ is a weak sub-solution (resp. super-solution) of (1), then $v=f(u)$ is a weak sub-solution (resp. super-solution) of (1). 


\section{The Moser method}

In this section we prove Theorem 1 . We first recall that, in the case of homogeneous Lie groups, it is not restrictive to consider the unit cylinder $R_{1}$ since the transformations of the form

$$
\zeta \longmapsto z_{0} \circ \delta_{r}(\zeta), \quad r>0, z_{0} \in \mathbb{R}^{N+1}
$$

preserve the class of differential equations considered. In our setting, we rely on the following result.

Lemma 5 The function $u$ is a weak solution of (1) in the cylinder $R_{r}\left(z_{0}\right)$ if and only if $v$ defined by

$$
v(\zeta)=u\left(z_{0} \circ \delta_{r}(\zeta)\right), \quad \zeta \in R_{1}
$$

is a solution to the equation

$$
\widetilde{L}_{r} v=\operatorname{div}(\widetilde{A} D v)+Y_{r} v=0, \quad \text { in } \mathrm{R}_{1},
$$

where $\widetilde{A}(\zeta)=A\left(z_{0} \circ \delta_{r}(\zeta)\right)$ satisfies hypothesis [H.2] with the same constant $\mu$ as $A$, and $Y_{r}$ is defined in (25).

Proof Since $Y_{r} \circ \delta_{r}=r^{2} \delta_{r} \circ Y$ and $Y$ is $\ell_{z_{0}}$-invariant, we have that

$$
Y_{r} \circ \delta_{r} \circ \ell_{z_{0}}=r^{2} \delta_{r} \circ \ell_{z_{0}} \circ Y
$$

or, more explicitly,

$$
Y_{r} v(\zeta)=Y_{r}^{(\zeta)} u\left(z_{0} \circ \delta_{r}(\zeta)\right)=r^{2}\left(Y^{(\zeta)}\left(u \circ \ell_{z_{0}}\right)\right)\left(\delta_{r}(\zeta)\right)=r^{2}(Y u)\left(z_{0} \circ \delta_{r}(\zeta)\right)
$$

where the superscript in $Y_{r}^{(\zeta)}$ indicates that we are differentiating w.r.t. the variable $\zeta$. On the other hand, recalling (13) and (18), we clearly have

$$
D_{m_{0}} v(\zeta)=D_{m_{0}}^{(\zeta)} u\left(z_{0} \circ \delta_{r}(\zeta)\right)=r\left(D_{m_{0}} u\right)\left(z_{0} \circ \delta_{r}(\zeta)\right)
$$

Thus we deduce $\widetilde{L}_{r} v(\zeta)=r^{2}(L u)\left(z_{0} \circ \delta_{r}(\zeta)\right)$ and the thesis follows.

Lemma 6 There exists a constant $\bar{c} \in] 0,1[$ such that

$$
z \circ_{r} R_{\bar{c}(1-\varrho)} \subseteq R_{1}
$$

for every $r \in[0,1], \varrho \in] 0,1\left[\right.$ and $z \in R_{\varrho}$. 
Proof Let $\varrho \in] 0,1\left[\right.$. By the expression (18) of the dilations $\left(\delta_{\lambda}\right)$, we see that

$$
R_{\varrho} \subseteq\left\{(x, t) \in \mathbb{R}^{N+1}|| x|<\varrho,| t \mid<\varrho^{2}\right\}
$$

Then the thesis is a consequence of the following inclusion: there exists a positive constant $c$ such that

$$
z \circ \circ_{\varepsilon} \subseteq\left\{(\xi, \tau)|| x-\xi|<c \varepsilon,| t-\tau \mid<(c \varepsilon)^{2}\right\}
$$

for every $\left.z \in R_{\varrho}, \varrho, \varepsilon \in\right] 0,1\left[\right.$. Indeed, if we choose $\varepsilon \leq \frac{1-\varrho}{c}$, we get

$$
z \circ_{r} R_{\varepsilon} \subseteq R_{1}, \quad \forall z \in R_{\varrho}
$$

and this shows (57) with $\bar{c}=c^{-1}$.

We are left with the proof of (58). If $\zeta=(\xi, \tau) \in z \circ_{r} R_{\varepsilon}$ then

$$
\zeta=z \circ_{r} \bar{z}=\left(\bar{x}+E_{r}(\bar{t}) x, t+\bar{t}\right)
$$

for some $\bar{z} \in R_{\varepsilon}$. Hence $|\tau-t|=|\bar{t}|<\varepsilon^{2}$, and

$$
|\xi-x|=\left|\bar{x}+\left(E_{r}(\bar{t})-E_{r}(0)\right) x\right| \leq|\bar{x}|+|\bar{t}| \max _{|r|,|s| \leq 1}\left\|E_{r}^{\prime}(s)\right\| \leq c \varepsilon
$$

where $c=1+\max _{|r|,|s| \leq 1}\left\|E_{r}^{\prime}(s)\right\|$.

As a consequence of the above lemmas, we only consider the unit cylinder in the proof of Theorem 1 and prove the claim for the operators of the form $\widetilde{L}_{r}$. We point out that, since its principal part is $K_{r}$, we use the group law " $\circ_{r}$ ". Theorem 1 is a consequence of the following uniform (in $r$ ) Caccioppoli and Sobolev type inequalities.

Theorem 3 (Caccioppoli type inequalities) Let $u$ be a non-negative weak solution of (56) for a given $r \in[0,1]$. Let $p \in \mathbb{R}, p \neq 0, p \neq \frac{1}{2}$ and let $\varrho, \bar{\varrho}$ be such that $\frac{1}{2} \leq \varrho<\bar{\varrho} \leq 1$. If $u^{p} \in L^{2}\left(R_{\varrho}\right)$ then $D_{m_{0}} u^{p} \in L^{2}\left(R_{\varrho}\right)$ and there exists a constant $c$, only dependent on the homogeneous dimension $Q$, such that

$$
\left\|D_{m_{0}} u^{p}\right\|_{L^{2}\left(R_{\varrho}\right)} \leq \frac{c \sqrt{\mu(\mu+\varepsilon)}}{\varepsilon(\bar{\varrho}-\varrho)}\left\|u^{p}\right\|_{L^{2}\left(R_{\bar{\varrho}}\right)}, \quad \text { where } \varepsilon=\frac{|2 p-1|}{4 p} .
$$

Theorem 4 (Sobolev type inequalities) Let $v$ be a non-negative weak solution of (56), for a given $r \in[0,1]$. Then $v \in L_{\mathrm{loc}}^{2 \kappa}\left(R_{1}\right), \kappa=1+\frac{2}{Q}$, and there exists a constant $c$, only dependent on $Q$ and $\mu$, such that

$$
\|v\|_{L^{2 \kappa}\left(R_{\varrho}\right)} \leq \frac{c}{\bar{\varrho}-\varrho}\left(\|v\|_{L^{2}\left(R_{\bar{\varrho}}\right)}+\left\|D_{m_{0}} v\right\|_{L^{2}\left(R_{\bar{\varrho}}\right)}\right)
$$

for every $\varrho, \bar{\varrho}$ with $\frac{1}{2} \leq \varrho<\bar{\varrho} \leq 1$. 
The proof of above estimates can be straightforwardly accomplished proceeding as in Theorems 3.1 and 3.3 in [28], by using the potential estimates of the previous section, and therefore is omitted. We are now in position to prove Theorem 1.

Proof of Theorem 1 Let $u$ be a positive solution to $L u=0$ in $R_{r}\left(z_{0}\right)$. By Lemma 5, the function $v(\zeta)=u\left(z_{0} \circ \delta_{r}(\zeta)\right)$, is a solution to (56) in $R_{1}$. We first prove that there exists a positive constant $c$ such that

$$
\sup _{R_{\varrho / r}} v^{p} \leq \frac{c}{(1-\varrho / r)^{Q+2}} \int_{R_{1}} v^{p}
$$

then, by the change of variable (55), we obtain (10), since

$$
\int_{R_{1}} u^{p}\left(z_{0} \circ \delta_{r}(\zeta)\right) d \zeta=\frac{1}{r^{Q+2}} \int_{R_{r}} u^{p}\left(z_{0} \circ w\right) d w=\frac{1}{r^{Q+2}} \int_{R_{r}\left(z_{0}\right)} u^{p}(z) d z,
$$

by Remark 2 .

In order to prove (61) it is sufficient to set $\theta=\bar{c}(1-\varrho / r)$, where $\bar{c}$ is the constant in Lemma 6, and to prove that

$$
\sup _{z \circ_{r} R_{\frac{\theta}{2}}} v^{p} \leq \frac{c \bar{c}^{Q+2}}{\theta^{Q+2}} \int_{z \circ_{r} R_{\theta}} v^{p}
$$

for every $z \in R_{\varrho / r}$.

By Lemma 5, the function $w(\zeta)=v\left(z \circ_{r} \delta_{\theta}(\zeta)\right)$ is a solution of

$$
\bar{L}_{r \theta} w=\operatorname{div}(\bar{A} D w)+Y_{r \theta} w=0, \quad \text { in } R_{1},
$$

where $\bar{A}(\zeta)=\widetilde{A}\left(z \circ \delta_{r} \delta_{\theta}(\zeta)\right)$ satisfies hypothesis [H.2] with the same constant $\mu$ as $A$. Hence, by a change of variables analogous to that in (62), in order to prove (63), it is sufficient to show that there exists a positive constant $c_{1}$, only depending on the constant $\mu$ in hypothesis [H.2] and on the matrix $B$, such that

$$
\sup _{R_{\frac{1}{2}}} u^{p} \leq c_{1} \int_{R_{1}} u^{p}
$$

for every positive solution $u$ of $\bar{L}_{\sigma} u=0$ : in particular, we emphasize that $c_{1}$ does not depend on $\sigma=r \theta \in[0,1]$.

We next prove (64). We first consider the case $p>0$ which is technically more complicated. Combining Theorems 3 and 4, we obtain the following estimate: if $q, \delta>0$ verify the condition $|q-1 / 2| \geq \delta$, then there exists a positive constant $c_{\delta}=c(\delta, Q, \mu)$, such that

$$
\left\|u^{q}\right\|_{L^{2 \kappa}\left(R_{\varrho}\right)} \leq \frac{c_{\delta}}{(r-\varrho)^{2}}\left\|u^{q}\right\|_{L^{2}\left(R_{r}\right)}
$$

for every $\varrho, r, \frac{1}{2} \leq \varrho<r \leq 1$, and $\sigma \in[0,1]$, where $\kappa=1+\frac{2}{Q}$. 
Fixed a suitable $\delta>0$ as we shall specify later and $p>0$, we iterate inequality (65) by choosing

$$
\varrho_{n}=\frac{1}{2}\left(1+\frac{1}{2^{n}}\right), \quad p_{n}=\frac{p \kappa^{n}}{2}, \quad n \in \mathbb{N} \cup\{0\} .
$$

We set $v=u^{\frac{p}{2}}$. If $p>0$ is such that

$$
\left|p \kappa^{n}-1\right| \geq 2 \delta, \quad \forall n \in \mathbb{N} \cup\{0\},
$$

by (65), we obtain

$$
\left\|v^{\kappa^{n}}\right\|_{L^{2 \kappa}\left(R_{\varrho_{n+1}}\right)} \leq \frac{c_{\delta}}{\left(\varrho_{n}-\varrho_{n+1}\right)^{2}}\left\|v^{\kappa^{n}}\right\|_{L^{2}\left(R_{\varrho n}\right)}, \quad \forall n \in \mathbb{N} \cup\{0\}
$$

Since

$$
\left\|v^{\kappa^{n}}\right\|_{L^{2 \kappa}}=\left(\|v\|_{L^{2 \kappa^{n+1}}}\right)^{\kappa^{n}} \text { and }\left\|v^{\kappa^{n}}\right\|_{L^{2}}=\left(\|v\|_{L^{2 \kappa^{n}}}\right)^{\kappa^{n}}
$$

we can rewrite (67) in the form

$$
\|v\|_{L^{2 \kappa^{n+1}}\left(R_{\varrho_{n+1}}\right)} \leq\left(\frac{c_{\delta}}{\left(\varrho_{n}-\varrho_{n+1}\right)^{2}}\right)^{\frac{1}{\kappa^{n}}}\|v\|_{L^{2 \kappa^{n}}\left(R_{\varrho n}\right)} .
$$

Iterating this inequality, we obtain

$$
\|v\|_{L^{2 \kappa^{n+1}}\left(R_{\varrho_{n+1}}\right)} \leq \prod_{j=0}^{n}\left(\frac{c_{\delta}}{\left(\varrho_{j}-\varrho_{j+1}\right)^{2}}\right)^{\frac{1}{\kappa^{j}}}\|v\|_{L^{2}\left(R_{1}\right)}
$$

and letting $n$ go to infinity, we get

$$
\sup _{R_{\frac{1}{2}}} v \leq \bar{c}\|v\|_{L^{2}\left(R_{1}\right)}
$$

where

$$
\bar{c}=\prod_{j=0}^{\infty}\left(\frac{c_{\delta}}{\left(\varrho_{j}-\varrho_{j+1}\right)^{2}}\right)^{\frac{1}{\kappa^{j}}},
$$

is a finite constant, dependent on $\delta$. Thus, we have proved (64) with $c_{1}=\bar{c}^{2}$, for every $p>0$ which verifies condition (66). 
We now make a suitable choice of $\delta>0$, only dependent on the homogeneous dimension $Q$, in order to show that (64) holds for every positive $p$. We remark that, if $p$ is a number of the form

$$
p_{m}=\frac{\kappa^{m}}{\kappa+1}, \quad m \in \mathbb{Z},
$$

then (66) is satisfied with $\delta=(2 Q+2)^{-1}$, for every $m \in \mathbb{Z}$. Therefore (64) holds for such a choice of $p$, with $c_{1}$ only dependent on $Q, \mu$. On the other hand, if $p$ is an arbitrary positive number, we consider $m \in \mathbb{Z}$ such that

$$
p_{m}=\frac{\kappa^{m}}{\kappa+1} \leq p<p_{m+1} .
$$

Hence, by (64), we have

$$
\sup _{R_{\frac{1}{2}}} u \leq\left(c_{1} \int_{R_{1}} u^{p_{m}}\right)^{\frac{1}{p_{m}}} \leq c_{1}^{\frac{1}{p_{m}}}\left(\int_{R_{1}} u^{p}\right)^{\frac{1}{p}}
$$

so that, by (68), we obtain

$$
\sup _{R_{\frac{1}{2}}} u^{p} \leq c_{1}^{\frac{p}{p_{m}}} \int_{R_{1}} u^{p} \leq c_{1}^{\kappa} \int_{R_{1}} u^{p} .
$$

This concludes the proof of (64) for $p>0$.

We next consider $p<0$. In this case, assuming that $u \geq u_{0}$ for some positive constant $u_{0}$, estimate (10) can be proved as in the case $p>0$ or even more easily since condition (65) is satisfied for every $p<0$. On the other hand, if $u$ is a nonnegative solution, it suffices to apply (10) to $u+\frac{1}{n}, n \in \mathbb{N}$, and to let $n$ go to infinity, by the monotone convergence theorem.

Proceeding as in the proof of Theorem 1, we obtain the following result

Corollary 2 Let $u$ be a non-negative weak sub-solution of (1) in $\Omega$. Let $z_{0} \in \Omega$ and $r, \varrho, \frac{1}{2} \leq \varrho<r \leq 1$, such that $R_{r}\left(z_{0}\right) \subseteq \Omega$. Then we have

$$
\begin{aligned}
& \sup _{R_{\varrho}\left(z_{0}\right)} u \leq\left(\frac{c}{(r-\varrho)^{Q+2}} \int_{R_{r}\left(z_{0}\right)} u^{p}\right)^{\frac{1}{p}}, \quad \forall p \geq 1, \\
& \inf _{R_{\varrho}\left(z_{0}\right)} u \geq\left(\frac{c}{(r-\varrho)^{Q+2}} \int_{R_{r}\left(z_{0}\right)} u^{p}\right)^{\frac{1}{p}}, \quad \forall p<0,
\end{aligned}
$$

with $c=c(Q, \mu)$. Estimate (70) is meaningful only when $u^{p} \in L^{1}\left(R_{r}\left(z_{0}\right)\right)$. 
Remark 3 Estimates (69) and (70) are a key point in the Moser's proof of the Harnack inequality. As stated in the introduction, we aim to adapt the Moser's method by proving a suitable Poincaré estimate in a future study.

We close this section by proving the local boundedness of weak solutions to (1).

Proof of Corollary 1 We consider a sequence $\left(g_{n}\right)_{n \in \mathbb{N}}$ in $C^{\infty}(\mathbb{R},[0,+\infty[)$ with the following properties:

$$
g_{n}(s) \downarrow \max (0, s), \quad s \in \mathbb{R}, \quad \text { as } n \rightarrow \infty,
$$

and, for every $n \in \mathbb{N}, g_{n}$ is a monotone increasing, convex function which is linear out of a fixed compact set. By Lemma $4,\left(g_{n}(u)\right)$ and $\left(g_{n}(-u)\right)$ are sequences of non-negative sub-solutions of $L$, which converge to $u^{+}=\max (0, u)$ and $u^{-}=$ $\max (0,-u)$, respectively. Thus, the thesis follows applying (69) of Corollary 2 to $g_{n}(u), g_{n}(-u)$ and passing at limit as $n$ goes to infinity.

Proof of Proposition 1 As in [28], we follow the lines of the proof of Theorem 1, by using the following two estimates:

$$
\left\|D_{m_{0}} u^{p}\right\|_{L^{2}\left(R_{\varrho}^{-}\right)} \leq \frac{c \sqrt{\mu(\mu+\varepsilon)}}{\varepsilon(r-\varrho)}\left\|u^{p}\right\|_{L^{2}\left(R_{r}^{-}\right)}, \quad \text { where } \varepsilon=\frac{|2 p-1|}{4 p}
$$

and

$$
\left\|u^{p}\right\|_{L^{2 \kappa}\left(R_{\varrho}^{-}\right)} \leq \frac{c}{r-\varrho}\left(\left\|u^{p}\right\|_{L^{2}\left(R_{r}^{-}\right)}+\left\|D_{m_{0}} u^{p}\right\|_{L^{2}\left(R_{r}^{-}\right)}\right)
$$

for every negative $p$ and for any $\varrho, r$ with $\frac{1}{2} \leq \varrho<r \leq 1$.

The Sobolev type inequality (72) can be proved exactly as Theorem 4 , since the fundamental solution $\Gamma(x, t, \xi, \tau)$ vanishes in the set $\{\tau>t\}$.

In order to prove the Caccioppoli type inequality (71) we we follow the method used in the proof of Theorem 3, by using $\varphi=u^{2 p-1} \psi^{2}$ as a test function, where $\chi_{n}(t)$ is defined as

$$
\chi_{n}(s)= \begin{cases}1, & \text { if } s \leq 0 \\ 1-n s, & \text { if } 0 \leq s \leq 1 / n \\ 0, & \text { if } s \geq 1 / n\end{cases}
$$

for every $n \in \mathbb{N}$. Then, by letting $n \rightarrow \infty$, we find

$$
\int_{R_{1}^{-}}\left(1-\frac{1}{2 p}\right) \psi^{2}\langle A D v, D v\rangle+\psi\langle A D v, D \psi\rangle+\frac{v^{2} \psi}{2} Y \psi \leq 0 .
$$

After that, we follow the same line used in the proof of Theorem 3 and we obtain (71). We refer to [28] for a more detailed proof of the analogous result in homogeneous Lie groups. 
Acknowledgments We thank E. Lanconelli for his interest in our work and an anonymous referee for some useful remarks that improved the readability of this paper.

\section{References}

1. Antonelli, F., Barucci, E., Mancino, M.E.: Asset pricing with a forward-backward stochastic differential utility. Econ. Lett. 72, 151-157 (2001)

2. Antonelli, F., Pascucci, A.: On the viscosity solutions of a stochastic differential utility problem. J. Differ. Equ. 186, 69-87 (2002)

3. Barucci, E., Polidoro, S., Vespri, V.: Some results on partial differential equations and Asian options. Math. Models Methods Appl. Sci. 11, 475-497 (2001)

4. Bhar, R., Chiarella, C.: The transformation of Heath-Jarrow-Morton models to Markovian systems. Eur. J. Finance 3, 1-26 (1997)

5. Bramanti, M., Cerutti, M.C., Manfredini, M.: $L^{p}$ estimates for some ultraparabolic operators with discontinuous coefficients. J. Math. Anal. Appl. 200, 332-354 (1996)

6. Cercignani, C.: The Boltzmann equation and its applications. Springer, New York (1988)

7. Citti, G., Pascucci, A., Polidoro, S.: Regularity properties of viscosity solutions of a non-Hörmander degenerate equation. J. Math. Pures Appl. 80(9), 901-918 (2001)

8. Desvillettes, L., Villani, C.: On the trend to global equilibrium in spatially inhomogeneous entropydissipating systems: the linear Fokker-Planck equation. Comm. Pure Appl. Math. 54, 1-42 (2001)

9. Di Francesco, M., Pascucci, A.: On the complete model with stochastic volatility by Hobson and Rogers. Proc. R. Soc. Lond. Ser. A Math. Phys. Eng. Sci. 460, 3327-3338 (2004)

10. Di Francesco, M., Pascucci, A.: On a class of degenerate parabolic equations of Kolmogorov type. AMRX Appl. Math. Res. Express, pp. 77-116 (2005)

11. Di Francesco, M., Polidoro, S.: Harnack inequality for a class of degenerate parabolic equations of Kolmogorov type. Adv. Diff. Equ. 11, 1261-1320 (2006)

12. Eidelman, S.D., Ivasyshen, S.D., Malytska, H.P.: A modified Levi method: development and application. Dopov. Nats. Akad. Nauk Ukr. Mat. Prirodozn. Tekh. Nauki 5, 14-19 (1998)

13. Folland, G.B.: Subelliptic estimates and function spaces on nilpotent Lie groups. Ark. Mat. 13, 161-207 (1975)

14. Heath, D.C., Jarrow, R.A., Morton, A.: Bond pricing and the term structure of interest rates. Econometrica 60, 77-106 (1992)

15. Hobson, D.G., Rogers, L.C.G.: Complete models with stochastic volatility. Math. Finance 8, 27-48 (1998)

16. Hörmander, L.: Hypoelliptic second order differential equations. Acta Math. 119, 147-171 (1967)

17. Il'in, A.M.: On a class of ultraparabolic equations. Dokl. Akad. Nauk SSSR 159, 1214-1217 (1964)

18. Kupcov, L.P.: The fundamental solutions of a certain class of elliptic-parabolic second order equations. Differencial'nye Uravnenija 8, pp. 1649-1660, 1716 (1972)

19. Lanconelli, E., Polidoro, S.: On a class of hypoelliptic evolution operators. Rend. Sem. Mat. Univ. Politec. Torino 52, 29-63 (1994). Partial differential equations, II (Turin, 1993)

20. Lifschitz, E.M., Pitaevskii, L.P.: Teoreticheskaya fizika ("Landau-Lifshits"). Tom 10, "Nauka", Moscow Fizicheskaya kinetika (Physical kinetics) (1979)

21. Lions, P.-L.: On Boltzmann and Landau equations. Philos. Trans. R. Soc. Lond. Ser. A 346, 191-204 (1994)

22. Lunardi, A.: Schauder estimates for a class of degenerate elliptic and parabolic operators with unbounded coefficients in $\mathbb{R}^{N}$. Ann. Scuola Norm. Sup. Pisa Cl. Sci. 24(4), 133-164 (1997)

23. Manfredini, M.: The Dirichlet problem for a class of ultraparabolic equations. Adv. Diff. Equ. 2, 831-866 (1997)

24. Manfredini, M., Polidoro, S.: Interior regularity for weak solutions of ultraparabolic equations in divergence form with discontinuous coefficients. Boll. Unione Mat. Ital. Sez. B Artic. Ric. Mat. 1(8), 651-675 (1998)

25. Morbidelli, D.: Spazi frazionari di tipo Sobolev per campi vettoriali e operatori di evoluzione di tipo Kolmogorov-Fokker-Planck, Tesi di Dottorato di Ricerca, Università di Bologna, Bologna (1998)

26. Moser, J.: A Harnack inequality for parabolic differential equations. Comm. Pure Appl. Math. 17, 101-134 (1964) 
27. Moser, J.: On a pointwise estimate for parabolic differential equations. Comm. Pure Appl. Math. 24, 727-740 (1971)

28. Pascucci, A., Polidoro, S.: The Moser's iterative method for a class of ultraparabolic equations. Commun. Contemp. Math. 6, 395-417 (2004)

29. Polidoro, S.: On a class of ultraparabolic operators of Kolmogorov-Fokker-Planck type. Matematiche (Catania) 49, 53-105 (1994)

30. Polidoro, S.: A global lower bound for the fundamental solution of Kolmogorov-Fokker-Planck equations. Arch. Ration. Mech. Anal. 137, 321-340 (1997)

31. Polidoro, S., Ragusa, M.A.: Sobolev-Morrey spaces related to an ultraparabolic equation. Manuscr. Math. 96, 371-392 (1998)

32. Risken, H.: The Fokker-Planck equation: Methods of solution and applications, 2nd edn. Springer, Berlin (1989)

33. Ritchken, P., Sankarasubramanian, L.: Volatility structures of forward rates and the dynamics of term structure. Math. Finance 5, 55-72 (1995)

34. Rothschild, L.P., Stein, E.M.: Hypoelliptic differential operators and nilpotent groups. Acta Math. 137, 247-320 (1976)

35. Šatyro, J.I.: The smoothness of the solutions of certain degenerate second order equations. Mat. Zametki 10, 101-111 (1971)

36. Weber, M.: The fundamental solution of a degenerate partial differential equation of parabolic type. Trans. Am. Math. Soc. 71, 24-37 (1951) 